\section{Fibre Optics Research at Battelle-Geneva}

\author{
A. L. Harmer, Battelle-Geneva
}

Battelle-Geneva is one of the five divisions of Battelle Memorial Institute which was founded in Columbus, Ohio, in 1923 by Gordon Battelle. In his will, Gordon Battelle left a part of his estate to found an Institute $« . .$. for the purpose of education in connection with the encouragement of creative and research work and the making of discoveries and inventions....". Today this Institute, combining the Battelle Research Laboratories with a total staff of 7100 people, is amongst the largest independent research organizations in the world.

Battelle-Geneva, created in 1953, employs some 400 people, nearly 300 of whom are researchers and technicians representing more than 20 different nationalities. Research is concentrated into three specialized areas which are adapted to the needs of industry: Industrial Technology, Toxicology and Biosciences, and Applied Economics. In contrast with the other divisions, Battelle-Geneva works essentially for private industry. Research is funded by sponsors, on an individual or group basis, usually to solve specific problems or to develop approaches and ideas suggested within Battelle.

Several fields in the Battelle-Geneva laboratories have a high content of physics, optics and electronics research. As typical examples one can cite flash spectrophotometry for biomedical sample analysis, UV gas lasers, solar energy panels, displays, electronic signal analysis and actuator engineering. This article covers only the research in fibre optics and seeks to convey, by a few chosen examples with a physics background, the way in which the work in the laboratories combines a response to industrial needs with a creative contribution from individual researchers, within the context of the normal evolution of research.

\section{ORIGINS OF MODERN FIBRE OPTICS}

Modern fibre optics can be seen as a maturing technology which, after only a single decade of exciting research and development, has already been applied to many telecommunications and industrial systems. To date, public telecommunications bodies in many countries have carried out field trials on high-bandwidth, fibreoptic links and are now installing long distance, point-to-point systems between major cities. This remarkable progress has been the result of a convergence of research results from different subjects and a combination of diverse technologies, such as glass chemistry, III-V semiconductor physics, micro-mechanical engineering, and sophisticated mathematical modelling of guided-wave light propagation.

In the late 1960s, world-wide research focused on two outstanding problems: the structure and fabrication of a low-loss fibre, and a suitable (solid-state) source. Battelle became involved in both. Since 1966, gallium arsenide has been produced in our laboratories, for light emitting diodes. The material is high purity and dislocation-free which is of particular importance for long-life devices. It has been supplied under contract to the Telecommunication Headquarters of the British Post Office 1). In 1971 as a result of a strong background in glass research and in thin film fabrication using chemical vapour deposition (CVD), Battelle began experiments on the fabrication of optical fibres by CVD, the work being supported by the Centre National d'Etudes des Telecommunications, France ${ }^{2}$ ). In the CVD technique, thin layers of silica are deposited on the inside of a silica tube. The refractive index of successive layers is increased by doping, building-up a concentric ring structure with a graded refractiveindex profile (Fig. $\Uparrow$ ). The tube is then collapsed to a solid rod or «preform», after which it is drawn into a fibre.

Low optical loss is achieved by producing material of very high purity. High bandwidth is obtained by minimizing the velocity difference between different modes (i.e. propagation paths) and this in turn is achieved by control of the graded refractive index profile. Also, by the appropriate choice of dopants the extent of dispersion of different wavelengths can be minimized. The availability of long lengths of fibre has allowed very accurate measurments to be made of absorption coefficients and has, in addition, stimulated the accurate determination of the refractive index dispersion of doped silica glasses. Absorption coefficients of less than $10^{-6} \mathrm{~cm}^{-1}$ can be measured in a kilometre length of fibre and this has led to a better fundamental understanding of the mechanisms at work.

\section{FIBRE PROPERTIES - Mechanical}

\section{Strength and Microbending Losses}

Material work in fibres is being continued here today with new techniques for pure glass preparation and fibre fabrication. Other fibre properties and characteristics are also studied, such as mechanical strength.

According to the classical Griffith theory, the strength of a glass (or brittle solid) is determined by the presence of surface flaws. The fracture strength $\sigma$ is inversely proportional to the square root of the radius of curvature $r$ of the flaw.

$\sigma=A r^{-1 / 2}$

where $A$ is a numerical constant.

Fig. 2 shows the cross-section of a silica fibre which failed at low strength during destructive traction testing. The crack originates at the flaw, travels across the smooth mirror region at subcritical crack velocity, accelerating to a limiting critical velocity (less than the Rayleigh wave velocity) which results in the hackle region and macroscopic cracks. The radius of each of these regions $\left(r_{m}\right.$ and $\left.r_{h}\right)$ is related to the fracture strength by the same Griffith formula with different mumerical constants $A$ (which can be derived from other studies). This allows accurate determination of the flaw size and, together with other techniques, e.g. chemical microprobe analysis, can identify the source of failure. In the photograph, the flaw is due to a carbon particle from contamination in the pulling furnace - a graphite resistance furnace operated at $2200^{\circ} \mathrm{C}$.

Studies have revealed the importance of clean pulling conditions and have resulted in techniques being developed to eliminate surface flaws and in the redesign of the furnace. Strengths of $30 \mathrm{GN} / \mathrm{m}^{2}$ for kilometre lengths of fibre are possible.

Surface flaws grow logarithmically with time causing a diminution in strength with age. We have also investigated methods to overcome this, such as producing a compressive stress on the fibre surface with a thin, low thermal expansion layer, or coating with a ceramic material.

Incidentally, the mechanism of fracture can be usefully exploited for the preparation of clean fibre ends (for connections or splices). By pre-determining the flaw size $(r)$ and tensile stress $(\sigma)$ the smooth mirror region can be made to traverse the whole fibre cross-section. The flaw is introduced deliberately, for example by contact with a heated wire, and the fibre pulled with a preset stress. A small fibre-breaking machine constructed in our laboratories has given good results and commercial devices are now available based on similar principles.

Microbending loss is another area of both research interest and of importance for industrial fibre use. From classical waveguide theory, using Maxwell's equations in a guiding structure, any distortion or curvature of a guide perturbs the electrical field pattern of the propagating light, leading to a change in the guided mode pattern. Loss occurs due to mode conver- 
sion on entering the curved region, and attenuation of the higher modes generated, either by a lossy cladding or by radiation to the outside of the guide.

Calculations of the radiation loss for the fundamental $\mathrm{HE}_{11}$ mode in a monomode fibre using the weak-guiding approximation are shown in Fig. 3, compared with the measured loss figures for a fibre distorted into a regular wave pattern. This is produced by compressing the fibre between two similar "combs" set out of phase with each other, the degree of overlap determining the curvature of the wave crests. Above cut-off, that is for long optical wavelengths, where the guide will only support a single mode ( $\mathrm{HE}_{11}$ mode), the loss is exponentially dependent on the radius of curvature. Below cut-off, the fibre is less sensitive to microbending. The structure in the measured curves arises from resonant mode coupling.

This area of study has important consequences for the design of monomode fibres whose core dimensions and doping are chosen to minimise loss, and also of cable packaging as distortions of the fibre within the jacket can give rise to increased losses of a few $\mathrm{dB} / \mathrm{km}$ due to microbending.

MICRO-OPTICS - Optical Components by Micro-mechanical Engineering

As in the development of semiconductor technology for device fabrication, experimental tools have to be invented and developed to allow handling and construction. This is particularly true of microoptics where attempts are made to produce miniature optical components with dimensions comparable with that of the fibre (100 $\mu \mathrm{m}$ or less). An example already cited is the method of obtaining a clean break in a fibre which exploits fracture phenomena.

Another example is the way microlenses can be made. A technique developed in our laboratories is hot forming with a $\mathrm{CO}_{2}$ laser whereby the surface tension of molten glass produces a natural lens shape. Perfect hemispherical lenses can be obtained

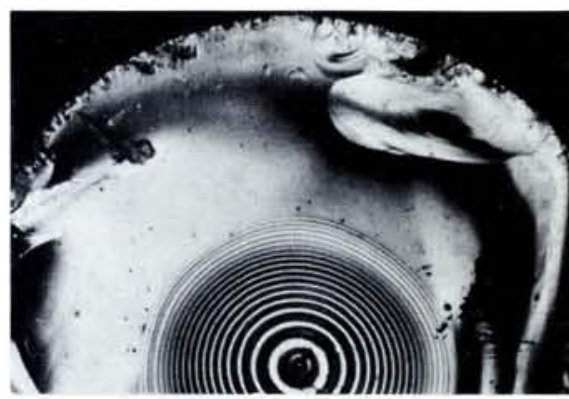

Fig. 1 - Section of an optical preform. The concentric rings, revealed by chemical etching, are the core region $14 \mathrm{~mm}$ diameter) made by depositing successive doped layers inside a silica tube.

on fibre ends by this method with a radius of curvature greater than $50 \mu \mathrm{m}$ down to a few microns. Such a lens structure is used to couple light from a laser diode into a fibre, resulting in an increased light gathering power of $4 \mathrm{~dB}$ or 2.5 times better than can be obtained by butt coupling of a plane-ended fibre.
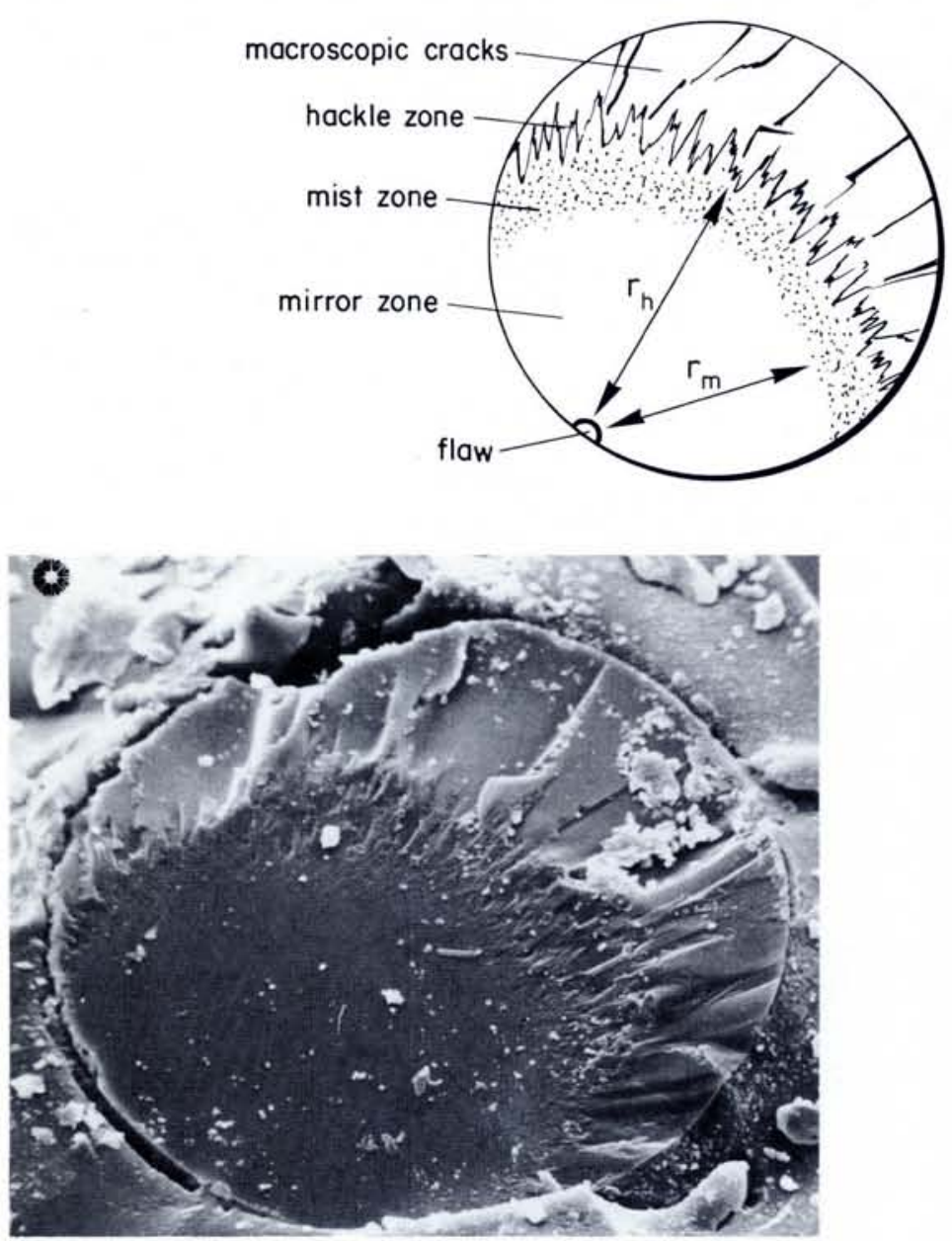

Fig. 2 - SEM picture (magnification $\times$ 650) of a low-strength fracture $\left(0.25 \mathrm{GN}\left(\mathrm{m}^{2}\right)\right.$ of a silica fibre (diameter $140 \mu \mathrm{m}$ ) with a soft silicone jacket. Measurement of mirror and hackle regions predicts a fracture strength of $0.27 \mathrm{GN} / \mathrm{m}^{2}$ with a flaw size of $1.5 \mu \mathrm{m}$. The origin of the flaw, a small carbon particle, is to be seen at the bottom edge of the fibre at the centre of the mirror zone. Debris on the fractured surface is from glass particles and dust.

\section{Universiteit van Amsterdam}

By mid-1981 the Department of Physics and Astronomy of the University of Amsterdam will have an opening for the position of

\section{(full)professor(m/f)}

\section{of theoretical physics.}

The appointed candidate will belong to the staff of the Institute for Theoretical Physics of the University of Amsterdam, where at present research is being conducted in the fields of high energy-and particle physics, kinetic theory of gases and plasmas, and statistical physics of phase transitions and critical phenomena.

The principle functions of the professor to be nominated will be:

- to perform, stimulate and supervise research in the field of statistical physics of many-body systems

- to inspire and stimulate co-operation between theoreticians and experimentalists in the fields of molecular physics and the physics of condensed matter

- to lecture for and to supervise the work of advanced undergraduate students and graduate students preparing a $\mathrm{Ph}$-D-thesis in one of the above-mentioned subjects.

Applications including a curriculum vitae, a list of publications and the names and addresses of a few referees, willing to provide information concerning personal and scientific qualifications, should be addressed before November 1, 1980 to the chairman of the Nomination Committee, Prof. Dr. J.Hijmans, Instituut voor Theoretische Fysica,

Valckenierstraat 65, 1018 XE Amsterdam, The Netherlands.

Please state vacancy reference number 3855 .

Those wishing to draw attention to potential candidates are also welcomed to contact the Committee Chairman not later than November 1, 1980. 


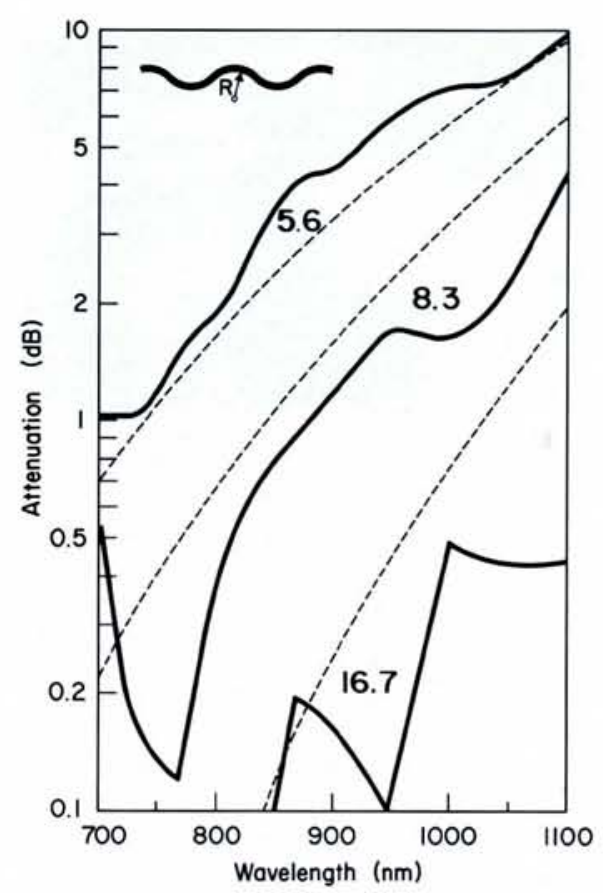

Fig. 3 - Theoretical wavelength dependence of the attenuation (dotted lines) compared to the measured microbending loss for a monomode fibre. Core size $10 \mu \mathrm{m}$, cut-off wavelength $820 \mathrm{~nm}$. Attenuation is for a fibre length of $20 \mathrm{~mm}$. The curves are for different radii of curvature, marked in $\mathrm{mm}$.

Another device we have built is a mechanical fibre switch in which a mobile fibre can move between two fixed fibres. This allows an optical channel to be commuted sequentially to two other optical channels. The alignment between the moving and fixed fibres is assured by precision $V$-grooves which reduce the optical coupling loss to less than $0.5 \mathrm{~dB}$. A large separation of the two fixed fibres (four times the fibre diameter) reduces crosstalk to less than $-75 \mathrm{~dB}$. The moving fibre is switched between the two fixed positions by a small bistable electromechanical actuator with a switching time of $2 \mathrm{~ms}$ and a power consumption of $0.6 \mathrm{~mJ}$. This device is useful in many applications, for example in instrumentation systems or in monitoring a system during installation by means of a switchable tap.

\section{FIBRE SENSORS -}

\section{New Types of Optical Transducers}

An interesting area of research, potentially important for industrial applications, is concerned with fibre sensors. Instrumentation nowadays uses mainly electronic transducers based on electronic or solidstate phenomena. With the invention of the low-loss optical fibre, all-optical instrumentation systems become possible in which an optical transducer produces an interaction of the physical variable with light which is conducted to and from the transducer by optical cables.
Transducers can conveniently be divided into two types: continuous (line) transducers and point transducers. In a continuous transducer, light is modulated along the length of the fibre by interaction with the physical variable (e.g. pressure, temperature), and the signal intensity is the integral over the interaction length, which is either the wavelength for a wavelike perturbation or the entire fibre length for a steady perturbation. A point transducer measures the interaction of the external physical field at a discrete point. Point transducers may either be constructed from a different material or the fibre may be treated to render it sensitive at a single point.

For continuous transducers, interferometry is a highly sensitive technique with many applications. For example, a simple gyroscope for inertial navigation systems can be constructed with, as sensitive element, a coil of single mode fibre in which counter circulating laser beams interfere with each other. Rotation of the reference frame changes the interference pattern which is read out by the electronic circuit. Dynamic pressure sensors use a similar arrangement - the phase shift of the light in the fibre being a function of the external pressure, the interaction length (acoustic wavelength) and the material piezo-optic coefficient. With a coil wound from a few metres of fibre, sensitivities higher than the most sensitive conventional pressure transducers can readily be obtained. Other applications, e.g. strain and temperature measurement are also possible, again with great sensitivity.

For point transducers, the inherent fibre characteristics may be modulated by an external variable. As a good example, we return to microbending effects, taking advantage of the extreme sensitivity of a fibre to small distortions or changes in its radius of curvature. Fig. 4 shows a prototype strain gauge, consisting of a fibre with periodic bends set so that changes in strain produce an increased optical attenuation. This device has also a very high sensitivity-a strain of $10^{-7}$ or a displacement of $1 \mathrm{~nm}$ is measurable. It shows almost no hysterisis $(<0.02 \%)$ and periodic cycling for more than $10^{7}$ strain cycles produces no change in the optical characteristics.

Other point transducers are being developed. A microprobe refractometer uses multiple reflections of glancingincidence light in a fibre to increase the sensitivity of refractive index measurements. A temperature gauge is based on phase changes in a material which can be incorporated into the fibre core. Many other devices, using a variety of optical phenomena (stress birefringence, electrooptic effect, Farady effect, radiationinduced luminescence) are also being investigated. In addition, novel modulation schemes (wavelength, polarisation and time-resolved modulation) are being devised to reduce instrumentation errors.

Further information on these and related subjects may be obtained directly from Battelle-Geneva on request.

\section{REFERENCES}

1. Baker J.F.C., Hart M., Halliwell M.A.G. and Heckingbottom $\mathrm{R}$.

Solid State Electronics, 19 (1976) 331.

2. Bouillie R. and Treheux M.

L'Echo des Recherches 81 (1975) 22.
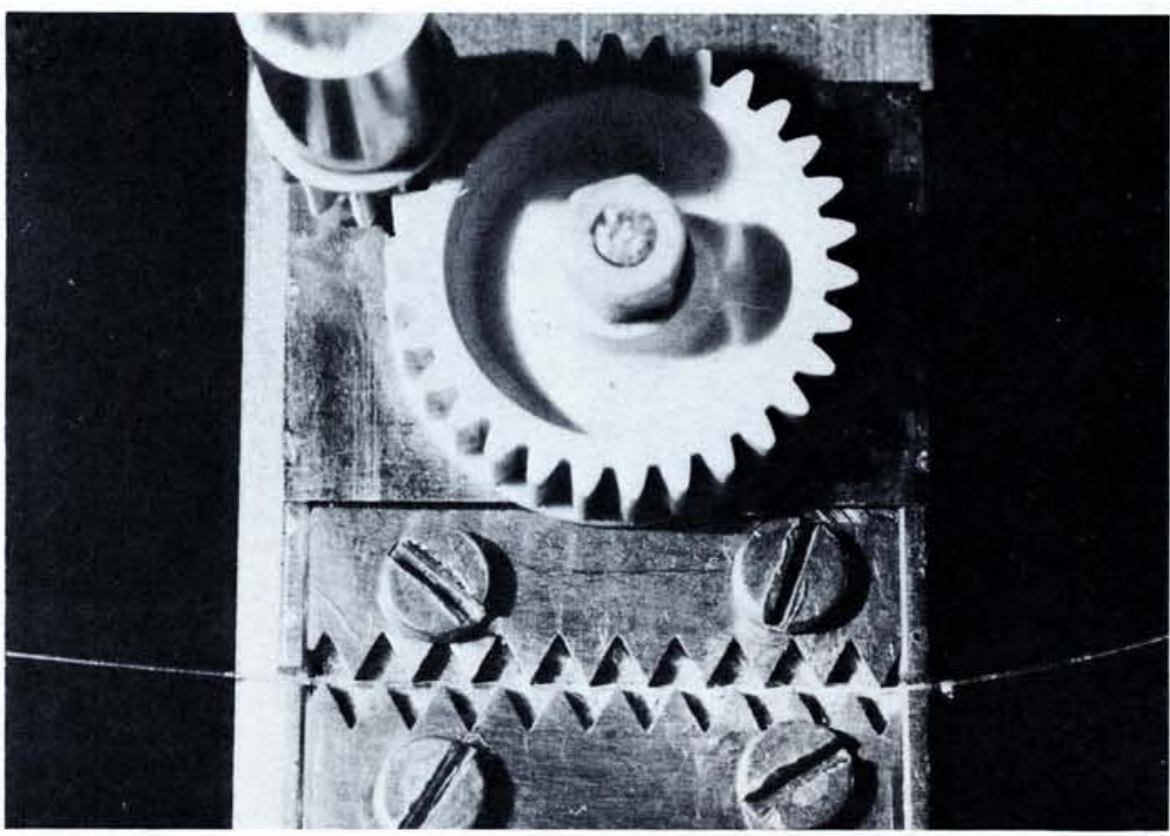

Fig. 4 - Prototype strain gauge. The fibre is clamped between saw-teeth. Light propagates from the left and undergoes progressive attenuation. The cogs are for regulating the initial strain setting. 